Publ. RIMS, Kyoto Univ.

13 (1977), 583-588

\title{
On an Application of the Averaging Method for Nonlinear Systems of Integro Differential Equations
}

\author{
By \\ D. D. Bainov* and G. H. Sarafova
}

Summary The present papes justifies a variant of the averaging method for a system of integıo difterential equations of a standard type, and finds an estimation tor proximity of the solutions of the considered system and its averaged system.

In paper [1] the averaging method for a system of ordinary differential equations of a standard type is justified. An estimation for proximity of the solutions of the initial and the averaged system is found.

In the present paper this method is applied to a nonlinear system of integro-differential equations of a standard type. An estimation for proximity of the solutions of the initial and its corresponding averaged system is found, using two of the schemes for averaging proposed in [2].

Consider the equation

$$
i=\varepsilon X\left(t, x, \int_{0}^{t} \varphi(t, s, x) d s\right)
$$

with initial condition

$$
x(0)=x_{0}
$$

where $x, X, \varphi \in \boldsymbol{R}^{n}$, and $\varepsilon>0$ is a small parameter.

Let the limit

$$
\lim _{T \rightarrow \infty} \frac{1}{T} \int_{0}^{T} X\left(t, x, \int_{0}^{t} \varphi(t, s, x) d s\right) d t=X_{0}(x)
$$

exist.

An averaged equation corresponding to (1) will be called the equation

Communicated by S. Hitotumatu, November 9, 1974.

* Department of Mathematics, University of Plovdiv, Paissji Hilendarski, Bulgaria. 


$$
\dot{\xi}=\varepsilon X_{0}(\xi)
$$

with initial condition

$$
\xi(0)=x_{0}
$$

The following theorem holds:

Theorem 1. Let the functions $X(t, x, y)$ and $\varphi(t, s, x)$ be defined and continuous in the domain $Q\left\{t, s \geqq 0, x \in \mathscr{D} \subset \boldsymbol{R}^{n}, y \in \mathbb{R}^{n}\right\}$, where the domain $\mathscr{D}$ is assumed to be open, and let, in this domain, the following conditions be satisfied:

1. There exists a constant $M$, such that $\|X(t, x, y)\| \leqq M$.

2. The functions $X(t, x, y)$ and $\varphi(t, s, x)$ satisfy the Lipschitz condition

$$
\begin{aligned}
& \left\|X\left(t, x^{\prime}, y^{\prime}\right)-X\left(t, x^{\prime \prime}, y^{\prime \prime}\right)\right\| \leqq \lambda\left\{\left\|x^{\prime}-x^{\prime \prime}\right\|+\left\|y^{\prime}+y^{\prime \prime}\right\|\right\}, \lambda=\text { const., } \\
& \left\|\varphi\left(t, s, x^{\prime}\right)-\varphi\left(t, s, x^{\prime \prime}\right)\right\| \leqq \mu(t, s)\left\|x^{\prime}-x^{\prime \prime}\right\| .
\end{aligned}
$$

3. $\frac{1}{t} \int_{0}^{t} d \tau \int_{0}^{\tau} \mu(\tau, s) d s \rightarrow 0, \quad t \rightarrow \infty$.

4. The limit (3) exists uniformly with respect to $x \in \mathscr{D}$.

5. The solution $\xi=\xi(t), \xi(0)=x_{0} \in \mathscr{D}$ of the Cauchy problem (4), (5) is defined for every $t \geqq 0$ and lies in $\mathscr{D}$ with some of its o-neighbourhoods.

Then, for each arbitrarily chosen, sufficiently large positive number $L>0$ there can be found such a number $\varepsilon_{0}>0$, that for $\varepsilon \in\left(0, \varepsilon_{0}\right]$ on the interval $0 \leqq t \leqq L \varepsilon^{-1}$ the following inequality would be satisfied:

$$
\|x(t)-\xi(t)\| \leqq e^{\lambda[L+\delta(\varepsilon)]}\{\lambda M L \delta(\varepsilon)+2 \psi(\varepsilon)+2 \sqrt{2 \lambda M L \psi(\varepsilon)(L+\delta(\varepsilon))}\}
$$

where

$$
\begin{gathered}
\psi(\varepsilon)=\sup _{0 \leqq \tau \leqq L}\left\{\sup _{\varepsilon \in \mathscr{D}}\left\|\int_{0}^{\tau / \varepsilon}\left[X\left(t, \xi, \int_{0}^{t} \varphi(t, s, \xi) d s\right)-X_{0}(\xi)\right] d t\right\|\right\}, \\
\delta(\varepsilon)=\sup _{0 \leqq \tau \leqq L} \tau \bar{\mu}_{0}\left(\frac{\tau}{\varepsilon}\right), \\
\bar{\mu}_{0}(t)=\frac{1}{t} \int_{0}^{t} d \tau \int_{0}^{\tau} \mu(\tau, s) d s
\end{gathered}
$$


Proof. We assume that $x(t) \in \mathscr{D}$ when $0 \leqq t \leqq L \varepsilon^{-1}$. For the difference $x(t)-\xi(t)$ there holds the integral representation

$$
\begin{aligned}
& x(t)-\xi(t)=\varepsilon \int_{0}^{t}\left[X\left(\tau, x(\tau), \int_{0}^{\tau} \varphi(\tau, s, x(s)) d s\right)\right. \\
&\left.-X\left(\tau, \xi(\tau), \int_{0}^{\tau} \varphi(\tau, s, \xi(s)) d s\right)\right] d \tau+\varepsilon \int_{0}^{t}[X(\tau, \xi(\tau), \\
&\left.\left.\int_{0}^{\tau} \varphi(\tau, s, \xi(s)) d s\right)-X\left(\tau, \xi(\tau), \int_{0}^{\tau} \varphi(\tau, s, \xi(\tau)) d s\right)\right] d \tau \\
&+ \varepsilon \int_{0}^{t}\left[X\left(\tau, \xi(\tau), \int_{0}^{\tau} \varphi(\tau, s, \xi(\tau)) d s\right)-X_{0}(\xi(\tau))\right] d \tau,
\end{aligned}
$$

whence the following estimation follows

$$
\text { (9) } \begin{gathered}
\|x(t)-\xi(t)\| \leqq \varepsilon \lambda \int_{0}^{t}\left\{\|x(\tau)-\xi(\tau)\|+\int_{0}^{\tau} \mu(\tau, s)\|x(s)-\xi(s)\| d s\right\} d \tau \\
+\varepsilon \lambda \int_{0}^{t} d \tau \int_{0}^{\tau} \mu(\tau, s)\|\xi(s)-\xi(\tau)\| d s+\varepsilon\left\|\int_{0}^{t} X_{1}(\tau, \xi(\tau)) d \tau\right\| .
\end{gathered}
$$

Here

$$
X_{1}(\tau, \xi(\tau))=X\left(\tau, \xi(\tau), \int_{0}^{\tau} \varphi(\tau, s, \xi(\tau)) d s\right)-X_{0}(\xi(\tau)) .
$$

The function $X_{1}(\tau, \xi(\tau))$ satisfies the Lipschitz condition. Indeed,

$$
\begin{aligned}
& \left\|X_{1}\left(\tau, \xi^{\prime}\right)-X_{1}\left(\tau, \xi^{\prime \prime}\right)\right\| \leqq \| X\left(\tau, \xi^{\prime}, \int_{0}^{\tau} \varphi\left(\tau, s, \xi^{\prime}\right) d s\right) \\
& -X\left(\tau, \xi^{\prime \prime}, \int_{0}^{\tau} \varphi\left(\tau, s, \xi^{\prime \prime}\right) d s\right)\|+\| X_{0}\left(\xi^{\prime}\right)-X_{0}\left(\xi^{\prime \prime}\right) \| \\
& \leqq \lambda\left\|\xi^{\prime}-\xi^{\prime \prime}\right\|+\lambda \int_{0}^{\tau} \mu(\tau, s)\left\|\xi^{\prime}-\xi^{\prime \prime}\right\| d s+\lim _{T \rightarrow \infty} \frac{1}{T} \int_{0}^{T} \| X\left(\tau, \xi^{\prime},\right. \\
& \left.\quad \int_{0}^{\tau} \varphi\left(\tau, s, \xi^{\prime}\right) d s\right)-X\left(\tau, \xi^{\prime \prime}, \int_{0}^{\tau} \varphi\left(\tau, s, \xi^{\prime \prime}\right) d s\right) \| d \tau \\
& \leqq \lambda\left\|\xi^{\prime}-\xi^{\prime \prime}\right\|+\lambda \int_{0}^{\tau} \mu(\tau, s)\left\|\xi^{\prime}-\xi^{\prime \prime}\right\| d s+\lambda\left\|\xi^{\prime}-\xi^{\prime \prime}\right\| \\
& +\lambda\left\|\xi^{\prime}-\xi^{\prime \prime}\right\| \lim _{T \rightarrow \infty} \frac{1}{T} \int_{0}^{T} d \tau \int_{0}^{\tau} \mu(\tau, s) d s \\
& =\left[2 \lambda+\lambda \mu_{0}(\tau)\right]\left\|\xi^{\prime}-\xi^{\prime \prime}\right\| .
\end{aligned}
$$

Here the notation $\mu_{0}(\tau)=\int_{0}^{\tau} \mu(\tau, s) d s$ is introduced. 
We estimate the last summand of (9) on the interval $0 \leqq t \leqq L \varepsilon^{-1}$. For this purpose we divide the interval into $m$ parts with the help of the points $t_{0}=0, t_{1}, \cdots, t_{m-1}, t_{m}=L \varepsilon^{-1}$ and we find

$$
\begin{gathered}
\left\|\varepsilon \int_{0}^{t} X_{1}(\tau, \xi(\tau)) d \tau\right\| \leqq\left\|\varepsilon \sum_{i=0}^{m-1} \int_{t_{i}}^{t_{i+1}}\left[X_{1}(\tau, \xi(\tau))-X_{1}\left(\tau, \xi\left(t_{i}\right)\right)\right] d \tau\right\| \\
+\left\|\varepsilon \sum_{i=0}^{m-1} \int_{t_{i}}^{t_{i+1}} X_{1}\left(\tau, \xi\left(t_{i}\right)\right) d \tau\right\|
\end{gathered}
$$

For the first summand on the right hand side of (10) we obtain the estimation

$$
\left\|\varepsilon \sum_{i=0}^{m-1} \int_{t_{i}}^{t_{i+1}}\left[X_{1}(\tau, \xi(\tau))-X_{1}\left(\tau, \xi\left(t_{i}\right)\right)\right] d \tau\right\| \leqq \frac{\lambda M L^{2}}{m}+\frac{\lambda M L}{m} \delta(\varepsilon)
$$

where $\delta(\varepsilon)$ is determined by $(7)$ and $\delta(\varepsilon) \rightarrow 0$ as $\varepsilon \rightarrow 0$.

From condition 4 of the theorem there follows the existence of the function

$$
\Phi(t)=\sup _{\xi \in \mathscr{D}}\left\|\frac{1}{t} \int_{0}^{t} X_{1}(\tau, \xi) d \tau\right\|, \quad \Phi(t) \rightarrow 0 \quad \text { as } \quad t \rightarrow \infty .
$$

Then

$$
\varepsilon\left\|\int_{0}^{t} X_{1}(\tau, \xi) d \tau\right\| \leqq \varepsilon t \bar{\Phi}(t) \leqq \sup _{0 \leqq \tau \leqq L} \tau \Phi\left(\frac{\tau}{\varepsilon}\right)=\psi(\varepsilon) \quad \phi(\varepsilon) \rightarrow 0 \quad \text { as } \quad \varepsilon \rightarrow 0
$$

For the second summand of (10) we get

$$
\left\|\varepsilon \sum_{i=0}^{m-1} \int_{t_{i}}^{t_{i+1}} \varphi\left(\tau, \xi\left(t_{i}\right)\right) d \tau\right\| \leqq 2 m \psi(\varepsilon) .
$$

From (10), (11), (12) there follows the estimation

$$
\left\|\varepsilon \int_{0}^{t} X_{1}(\tau, \xi(\tau)) d \tau\right\| \leqq \frac{\lambda M L^{2}}{m}+\frac{\lambda M L}{m} \delta(\varepsilon)+2 m \psi(\varepsilon) .
$$

From (9) and (13) we obtain

$$
\begin{gathered}
\|x(t)-\xi(t)\| \leqq \varepsilon \lambda \int_{0}^{t}\left\{\|x(\tau)-\xi(\tau)\|+\int_{0}^{\tau} \mu(\tau, s)\|x(s)-\xi(s)\| d s\right\} d \tau \\
+\lambda M L \delta(\varepsilon)+\frac{\lambda M L^{2}}{m}+\frac{\lambda M L}{m} \delta(\varepsilon)+2 m \psi(\varepsilon)
\end{gathered}
$$




$$
\|x(t)-\xi(t)\| \leqq\left[\lambda M L \delta(\varepsilon)+\frac{\lambda M L^{2}}{m}+\frac{\lambda M L}{m} \delta(\varepsilon)+2 m \psi(\varepsilon)\right] e^{\lambda[L+\delta(\varepsilon)]}
$$

whence we get the estimation

$$
\|x(t)-\xi(t)\| \leqq e^{\lambda[L+\delta(\varepsilon)]}[\lambda M L \delta(\varepsilon)+2 \psi(\varepsilon)+2 \sqrt{2 \lambda M L \psi(\varepsilon)[L+\delta(\varepsilon)]}] .
$$

The proof of the fact that $x(t) \in \mathscr{D}$ when $t \in\left[0, L \varepsilon^{-1}\right]$ is trivial. In this way Theorem 1 is proved.

Suppose that the limit

$$
\lim _{T \rightarrow \infty} \frac{1}{T} \int_{0}^{T} X\left(t, x, \int_{0}^{\infty} \varphi(t, s, x) d s\right) d t=X_{0}(x)
$$

exists.

Then the following theorem holds:

Theorem 2. Let the functions $X(t, x, y)$ and $\varphi(t, s, x)$ be defined and continuous in the domain $Q\left\{t, s \geqq 0, x \in \mathscr{D} \subset \boldsymbol{R}^{n}, y \in \mathbb{R}^{n}\right\}$ and let the following conditions be satisfied in this domain:

1. $\|X(t, x, y)\| \leqq M, \quad M=$ const .

2. $\left\|X\left(t, x^{\prime}, y^{\prime}\right)-X\left(t, x^{\prime \prime}, y^{\prime \prime}\right)\right\| \leqq \lambda\left\{\left\|x^{\prime}-x^{\prime \prime}\right\|+\left\|y^{\prime}-y^{\prime \prime}\right\|\right\}$

$$
\left\|\varphi\left(t, s, x^{\prime}\right)-\varphi\left(t, s, x^{\prime \prime}\right)\right\| \leqq \mu(t, s)\left\|x^{\prime}-x^{\prime \prime}\right\|
$$

3. $\frac{1}{t} \int_{0}^{t} d \tau \int_{0}^{\tau} \mu(\tau, s) d s \rightarrow 0, \quad t \rightarrow \infty ; \lambda=$ const .

4. The limit (14) exists uniformly with respect to $x \in \mathscr{D}$.

5. The solution $\xi=\xi(t), \xi(0)=x(0) \in \mathscr{D}$ of the averaged equation is defined for every $t \geqq 0$ and lies in $\mathscr{D}$ with some of its 0 -neighbourhoods.

6. $\lim _{t \rightarrow \infty} \frac{1}{t} \int_{0}^{t}\left\|\int_{0}^{\infty} \varphi(\tau, s, \xi(\tau)) d s\right\| d \tau=0$

7. $\left\|\int_{0}^{\infty} \varphi\left(t, s, x^{\prime}\right) d s-\int_{0}^{\infty} \varphi\left(t, s, x^{\prime \prime}\right) d s\right\| \leqq \nu\left\|x^{\prime}-x^{\prime \prime}\right\|, \quad \nu=$ const .

Then, for every $L>0$ there exists $\varepsilon_{0}>0$, such that when $0<\varepsilon \leqq \varepsilon_{0}$ on the interval $0 \leqq t \leqq L \varepsilon^{-1}$ the following inequality is fulfilled: 


$$
\begin{gathered}
\|x(t)-\xi(t)\| \leqq \\
e^{\lambda[L+\delta(\varepsilon)]}\left\{\lambda \gamma(\varepsilon)+\lambda M L \delta(\varepsilon)+2 \psi_{1}(\varepsilon)\right. \\
\left.+2 \sqrt{2 \lambda M L^{2} \psi_{1}(\varepsilon)(1+\nu)}\right\}
\end{gathered}
$$

where

$$
\begin{aligned}
& \phi_{1}(\varepsilon) \sup _{0 \leqq \tau \leqq L}\left\{\sup _{\xi \in \mathscr{D}}\left\|\int_{0}^{\tau / \varepsilon}\left[X\left(t, \xi, \int_{0}^{\infty} \varphi(t, s, \xi) d s\right)-X_{0}(\xi)\right] d t\right\|\right\}, \\
& \gamma(\varepsilon)=\sup _{0 \leqq \tau \leqq L} \tau F\left(\frac{\tau}{\varepsilon}\right), \quad F(t)=\frac{1}{t} \int_{0}^{t}\left\|\int_{\tau}^{\infty} \varphi(\tau, s, \xi(\tau)) d s\right\| d \tau .
\end{aligned}
$$

The proof of Theorem 2 is analogous to that of Theorem 1 .

\section{References}

[1] Besjes, J. G., On the asymptotic methods for non-linear differential equations, $J$. Mécanique, 8 (1969), N 3.

[2] Filatov, A. N., Metodi usrednenjia $v$ diferentsialnih i integro-diferentsialnih urav. nenijah. Izd. "FAN", Tashkent, 1971. 